\title{
El papel del docente reflexivo como estrategia del síndrome de Burnout en relación con las prácticas educativas*
}

\author{
Cristian Fernán Muñoz Muñoz ${ }^{* *}$, Claudia Milena Correa Otálvaro ${ }^{* * *}$
}

\section{RESUMEN}

Este artículo de investigación presenta los resultados de un estudio cuyo objetivo es identificar la relación entre el síndrome de Burnout y las prácticas educativas en docentes de primaria y secundaria. El diseño metodológico inicia con la identificación del nivel de prevalencia del síndrome; para tal fin se utilizó el inventario de Burnout de Maslash, el cual fue aplicado en una institución educativa. Los resultados indican una presencia del síndrome, con una prevalencia alta en el $12 \%$ de la población; media en el 70 \% y baja en el $18 \%$. Seguidamente, se realizó una entrevista a profundidad con el propósito de identificar, desde las experiencias de los docentes, aquellos elementos que son factores de riesgo en la aparición del síndrome. Las entrevistas fueron analizadas desde la teoría fundamentada de Corbin y Strauss (2001), en la cual se pueden identificar tres momentos: abierta, axial y selectiva. Los resultados fueron codificados en cuatro categorías: síntomas de agotamiento laboral, prácticas educativas como detonante del Burnout, sistema de afrontamiento desde las prácticas y afrontamiento por fuera de las prácticas. El artículo termina haciendo una profunda reflexión sobre la importancia del docente reflexivo como medio de afrontamiento de los factores de riesgo del Burnout.

Palabras clave: síndrome de Burnout, prácticas educativas, docente, docente reflexivo.

El artículo es resultado del proceso investigativo del trabajo de grado de la maestría en Educación de la Universidad Católica de Manizales. Cómo citar este artículo: Muñoz Muñoz, C.F. y Correa Otálvaro, C.M. (2014). El papel del docente reflexivo como estrategia del síndrome de Burnout en relación con las prácticas educativas. Hallazgos, 11(22), 385-401.

** Psicólogo, magíster en Educación de la Universidad Católica de Manizales. Psicoorientador del Colegio La Salle (Pereira), docente de la Universidad Minuto de Dios, docente de la Corporación de Estudios Tecnológicos del norte del Valle e investigador del grupo "Sujeto, Mente y Comunidad (Colombia)". E-mail: cristianfernam@hotmail.com

*** Pedagoga Infantil, estudiante en espera de grado de la maestría en Educación de la Universidad Tecnológica de Pereira. Joven Investigadora Colciencias, docente de la Corporación de Estudios Tecnológicos del norte del Valle y docente de básica primaria del Colegio La Presentación. E-mail: co_milena@hotmail.com 


\title{
The role of the reflexive teacher as a strategy for Burnout syndrome regarding teaching practices
}

\begin{abstract}
This research paper presents the results of a study aimed at identifying the relationship between burnout syndrome and educational practices in primary and secondary teachers. The methodology starts with the identification of the level of prevalence of the syndrome, for this purpose the Maslash Burnout Inventory, which was applied in an educational institution was used. The results indicate the presence of the syndrome, with a high prevalence in $12 \%$ of the population, on average, in $70 \%$ and low in $18 \%$. Next, a depth interviews in order to identify, from the experiences of teachers, those elements that are risk factors in the onset of the syndrome was performed. The interviews were analyzed from a grounded theory of Strauss and Corbin (2001) in which we can identify three stages: open, axial and selective. The results were coded into three categories: symptoms of job burnout, educational practices as a trigger of Burnout, coping system from outside practices and coping practices. The article ends by a deep reflection on the importance of reflective teaching as a means of coping with risk factors Burnout.
\end{abstract}

Keywords: Burnout syndrome, educational practices, teaching, reflective teaching. 


\section{O papel do professor reflexivo como uma estratégia da síndrome de Burnout em relação às práticas educativas}

\section{Resumo:}

Este artigo de investigação apresenta os resultados de um estudo cujo objetivo é identifica a relação entre a síndrome de Burmout e as práticas educacionais entre os professores de primeiro e segundo grau. $\mathrm{O}$ desenho metodológico inicia com a identificação do nível de prevalência da síndrome; para isso foi utilizado o inventário de Burnout de Maslash, que foi aplicado em uma instituição de ensino. Os resultados indicam uma presença da síndrome, com alta prevalência em $12 \%$ da população; média em $70 \%$ e $18 \%$ em baixo. Em seguida, foi feita uma entrevista em profundidade a fim de identificar, a partir das experiências dos professores, os elementos que são fatores de risco no aparecimento da síndrome. As entrevistas foram analisadas a partir da teoria fundamentada de Strauss e Corbin (2001), no qual podem-se identificar três momentos: aberta, axial e seletiva. Os resultados foram codificados em quatro categorias: sintomas de esgotamento no trabalho, práticas educativas como um gatilho para o Burnout, sistema de enfrentamento a partir das práticas e enfrentamento por fora das práticas. $\mathrm{O}$ artigo termina com uma reflexão profunda sobre a importância do docente reflexivo como um meio de afrontamento com os fatores de risco do Burnout.

Palavras-chave: Síndrome de Burnout, práticas educativas, professor, docente reflexivo. 


\section{INTRODUCCIÓN}

La realidad laboral de los docentes ha impuesto una serie de cambios y necesidades que han ido en detrimento de la salud de los colaboradores en las instituciones educativas. A partir de ello, el sujeto se ha visto sobreexpuesto a un continuo desgaste personal, haciéndolo propenso a padecer disfuncionalidades del orden físico, emocional y psicológico. Este hecho ha dado origen a nuevos problemas de salud, especialmente en personas cuyas actividades implican un servicio de tipo social (Gil y Peiró, 1999; Agudo, 2004; Aldrete, et al., 2003; Cordeiro, et al., 2003; El Sahili yKornhauser, 2010; Muñoz y Piernagorda, 2011).

La creciente preocupación por el bienestar organizacional ha permitido la investigación y conceptualización de los factores de riesgo en el ámbito laboral. En 1974 el psiquiatra Freudenberger introdujo el concepto de síndrome de Burnout (SB), al observar en un grupo de voluntarios que trabajaban con toxicómanos padecimientos de salud de modo progresivo, como pérdida de energía y agotamiento, episodios de ansiedad y depresión, desmotivación para su trabajo y agresividad con las personas. El SBo desgaste ocupacional es una metáfora referida al profesional "quemado", al trabajador emocionalmente exhausto y fatigado, cuyas manifestaciones emergen como respuesta al estrés crónico en el trabajo. Maslach y Jackson (1986) definen el SB como una manifestación comportamental del estrés laboral, se identifican tres dimensiones:

Agotamiento emocional (AE): el sujeto presenta un estado de agotamiento que repercute en el nivel comunicativo que establece con aquellos que se relaciona, y se desvincula así del mundo simbólico-afectivo. Se experimenta una sensación disipada y disminuida de la facultad de sentir; así, lo emocional se encuentra vacío y falto de energía (Unda, 2010; Yslado, Nuñez y Norabuena, 2010; Moreno et al., 2010).

Despersonalización (D): el sujeto presenta una alteración perceptual de sí mismo, se siente "separado" de los procesos mentales, experimentándose como un observador externo de sí. El nivel comunicativo intrapersonal se encuentra desestructurado, sin identidad, extraño a sí mismo, sin comprensión de lo real y desorientación en el tiempo y el espacio. El trato con los usuarios empieza a ser poco cercano, negativo y apático. El otro aparece como un algo y no como un alguien (Noyola, Padilla, 2010; Núñez, 2010; Gantiva, Jaimes y Villa, 2010; García, Sáez, Concepción y Bartolomé, 2000).

Falta de realización profesional (RP): el sujeto se siente estancado, en stand by, sin posibilidades percibidas de apertura en el ejercicio de su profesión, demostrando actitudes negativas hacia el rol profesional. El mundo simbólico, relacional y existencial de su profesión pierde su rumbo, y genera sentimientos de incompetencia, pérdida de eficacia y poca productividad (EscalanteIzeta2010; Ramírez, D'aubeterre, Álvarez, 2010; Rionda, Mares, 2011; Punceles y Tovar, 2011).

La práctica de la profesión docente presenta una serie de condiciones que facilitan la vulnerabilidad a presentar el SB. En la praxis docente se encuentran factores desencadenantes que desestabilizan la salud de los profesores, vinculados principalmente 
con el modo como se establecen interacciones comunicativas con el entorno, consigo mismo, alumnos, padres de familia y compañeros de trabajo. De esta manera, es un hecho común observar a los docentes adolecer de complicaciones de carácter somático, psicológico y emocional. De acuerdo con lo anterior y conscientes de esta realidad de la profesión educativa, se han venido realizando diversos estudios encaminados a la medición, prevención e intervención del SB (Moreno-Jiménez, Garrosa y González, 2000; Moriana y Herruzo, 2003; Salanova, Llorens y García, 2003; Restrepo, Colorado y Cabrera, 2006; Padilla, Gómez, Rodríguez, Dávila, Avella, Caballero y Vives, 2009; Muñoz, 2012).

El SB se presenta cuando el docente no posee las suficientes estrategias de afrontamiento que le permiten enfrentarse al estrés laboral crónico (Arata, 2008; Aguilar, Barros y Caro, 2011; y Correa, 2012; Díaz, López y Varela, 2012). La práctica educativa (PE) como macrosistema, vinculada a dinámicas y exigencias sociales, y como microsistema, experimentada como una realidad vital de interacciones y de subjetividades, se convierte en el espacio donde el docente encuentra los factores de riesgo o los mecanismos de superación de una realidad que lo lleva al enajenamiento y a la alienación (Mora, 2006; Perrenoud, 2006; Zabala, y Arnau, 2008; Zabala, 2008).

\section{Método}

El ciclo investigativo se encuentra evidenciado como una espiral ascendente, iniciado con la medición SB en docentes, desde un método cuantitativo de tipo transversal. Seguidamente, se realizó el reconocimiento e identificación de problemas en relación con su práctica docente, como son los factores de riesgo del estrés profesional. Para aproximarnos a las distintas posiciones con respecto al SB y la relación con las PE, se realizaron producciones narrativas (PN) de acuerdo con la propuesta de Balasch y Montenegro (2003). Dichas producciones consisten en la producción conjunta de un texto híbrido entre investigador y participante, y son generadas a través de sesiones de conversación sobre el tema de estudio, la producción de texto sobre dichas conversaciones por parte del investigador y la agencia de la persona participante sobre el texto, para modificarlo y hacerlo concordar gradualmente con su punto de vista.

\section{INSTRUMENTOS}

Para medir la prevalencia del SB, se aplicó el inventario de Burnout de Maslach (MBI), conformado por 22 ítems tipo Likert, con rango de 0 a 6 desde "nunca" a "diariamente", en tres categorías: AE, D, y RP (Maslach y Jackson, 1986). Seguidamente, se realizó una entrevista a profundidad acerca de las percepciones que los docentes tienen sobre la relación del SB y sus PE. Para el análisis posterior se utilizarán el diario de campo, las planeaciones del docente, el autoinforme docente y las grabaciones de audio y video de la clase, para identificar los factores de riesgo en las $\mathrm{PE}$,

\section{Población}

Estuvo compuesta por 16 docentes (100\% de la población) de básica primaria y secundaria de una institución educativa privada, de los cuales el $56 \%$ corresponde a mujeres y el $44 \%$, a hombres. El 56,7\% son casados; el 
$37,5 \%$, solteros y el 6,3\%, divorciados. El 37,5\% de los docentes están entre los 31 y 40 años; un $31,3 \%$, entre los 20 y 30 años, y los 41 y 50 años. Las horas laborales están ubicadas en tres categorías de donde el 43,8\% trabaja de 25 a 35 y de 36 a 40 horas semanales, y el $12,5 \%$ labora de 46 horas en adelante. En cuanto a los años de experiencia laboral, el $50 \%$ se encuentra entre 11 y 20 años de experiencia; el $43,8 \%$ entre 1 y 10 años y finalmente el $6,3 \%$ tiene más de 21 años de experiencia.

\section{AnÁlisis}

En la medición del SB, no hay puntaciones de corte a nivel clínico para medirse existencia, aunque se puede establecer qué puntuaciones altas en AE y D, y baja en RP definen su presencia. Los profesionales por encima del percentil 75 se clasificarán en padecimiento alto, entre el percentil 75 y 25 en medio y por debajo del percentil 25 en bajo (Maslach y Jackson, 1986).

Tabla 1. Percentiles

\begin{tabular}{rrrcc}
\hline & & Agotamiento emocional & Realización profesional & Despersonalización \\
\hline Percentiles & 25 & 14 & 33 & 6 \\
\hline 50 & 17 & 36 & 9 \\
\hline 75 & 20 & 37 & 10 \\
\hline
\end{tabular}

Fuente: elaboración propia

Las puntuaciones superiores a 20 en el componente AE muestran un nivel alto, el intervalo entre 15 y 17 corresponde a un nivel medio, y por debajo de 14 indican nivel bajo. En la subescala de D, las puntuaciones superiores a 10 se consideran de nivel alto; de 7 a 9, medio, y las inferiores a 6, bajo. Por último, en la subescala de RP, de 0 a 33 son de nivel alto; de 34 a 36, de nivel medio, y superior a 40, de nivel bajo. En la fase de análisis del MBI se utiliza el programa SSSP 19 para determinar el nivel de Burnoutde los docentes. Después de organizado el corpus documental de las entrevista, se inicia la fase de análisis e interpretación, para lo cual se utilizó el procedimiento de codificación y categorización de la información recogida. Este proceso está diseñado desde la teoría fundamentada de Corbin y Strauss (2001), en la cual se pueden identificar tres momentos: abierta, axial y selectiva.

\section{Resultados}

En la tabla 2 se registran los resultados de la sumatoria de los ítems de cada uno de los componentes del SB en los 16 docentes que diligenciaron el cuestionario. Podemos notar que existe una prevalencia alta de Burnout en un solo docente, el número cinco, donde los tres componentes refieren un grado alto de prevalencia: AE $\left(\sum=26\right), \mathrm{D}\left(\sum=10\right)$ y RP $\left(\sum=32\right)$. Por otro lado, un solo docente aparece con prevalencia baja del SB, el número nueve, presentando en los tres componentes puntuación ubicados en el percentil bajo $\mathrm{AE}\left(\sum=11\right)$, D $\left(\sum=5\right)$ y RP $\left(\sum=40\right)$. Finalmente, el resto de la población docente se encuentra en nivel medio, pues muchos de los componentes se ubican en este nivel de padecimiento. Cabe notar que varios docentes presentan uno o dos componentes en nivel alto del síndrome. De esta manera, en el AE 
se ubican cinco docentes en nivel alto de padecimiento; en la RP hay sietes docentes y en la D están seis docentes. Por otro lado, en el nivel medio hay nueve docentes en el componente AE; en RP hay tres docentes y en el componente de D están ubicados cinco docentes. Por último, en nivel bajo de padecimiento en AE hay dos personas; en el componente de RP se ubican siete docentes $\mathrm{y}$ finalmente en $\mathrm{D}$ hay cuatro docentes.

Tabla 2. Sumatoria $\left(\sum\right)$ componentes MBI del SB por docente

\begin{tabular}{cccc}
\hline $\mathbf{N}^{\circ}$ & Agotamiento emocional & Realización Personal & Despersonalización \\
\hline 1 & 12 & 36 & 5 \\
\hline 2 & 15 & 37 & 6 \\
\hline 3 & 10 & 36 & 5 \\
\hline 4 & 14 & 33 & 11 \\
\hline 5 & 26 & 32 & 10 \\
\hline 6 & 20 & 37 & 8 \\
\hline 7 & 14 & 33 & 10 \\
\hline 8 & 14 & 33 & 9 \\
\hline 9 & 11 & 40 & 5 \\
\hline 10 & 17 & 33 & 6 \\
\hline 11 & 20 & 37 & 9 \\
\hline 12 & 27 & 30 & 7 \\
\hline 13 & 17 & 37 & 10 \\
\hline 14 & 17 & 37 & 13 \\
\hline 15 & 16 & 36 & 10 \\
\hline 16 & 20 & 40 & \\
\hline
\end{tabular}

Fuente: elaboración propia

La tabla 3 nos presenta los datos descriptivos de la población diagnosticada con el MBI. Puede notarse que la media de los tres componentes se encuentra en un nivel medio de padecimiento del SB (AE, $\mathrm{M}=17,8 ; \mathrm{D}, \mathrm{M}=9,37 ; \mathrm{RP}, \mathrm{M}=35,44)$. El AE presenta una varianza y desviación típica alta.

Tabla 3. Prevalencia de Burnout en docentes de la institución educativa

\begin{tabular}{lccccccc}
\hline & N & Rango & Mínimo & Máximo & Media & Desv. Típ. & Varianza \\
\hline Agotamiento emocional & 16 & 17 & 10 & 27 & 17,8 & 4,829 & 23,317 \\
\hline Realización profesional & 16 & 10 & 30 & 40 & 35,44 & 2,828 & 7,996 \\
\hline Despersonalización & 16 & 8 & 5 & 13 & 9,37 & 2,446 & 5,983 \\
\hline N válido & 16 & & & & & & \\
\hline
\end{tabular}

Fuente: elaboración propia 
La tabla 4 presenta que el componente AE es mayor en personas solteras $(\mathrm{m}=17,33)$, ubicándose en nivel de padecimiento medio, mientras que los docentes casados se ubican en nivel bajo $(m=16,78)$, al igual que los docentes en unión libre $(\mathrm{m}=15)$. Así mismo, la $D$ es superior en solteros $(m=10,32)$ que en casados $(\mathrm{m}=7,67)$ y en docentes en unión libre $(m=6)$. Por otro lado, el nivel de RP es más favorable en docentes en unión libre $(m=37)$ que en solteros $(m=34,67)$ y casados $(\mathrm{m}=35,78)$.

Tabla 4. Prevalencia de Burnout según estado civil

\begin{tabular}{lccc}
\hline & Agotamiento emocional & Despersonalización & Realización Personal \\
\hline Casado & 16,78 & 7,67 & 35,78 \\
\hline Soltero & 17,33 & 10,32 & 34,67 \\
\hline Unión libre & 15,00 & 6,00 & 37,00 \\
\hline
\end{tabular}

Fuente: elaboración propia

Según la tabla 5, la media en el componente de $A E$ es superior en mujeres $(m=17,11)$ que en hombres $(\mathrm{m}=16,57)$, ubicando a las mujeres en un rango medio de padecimiento de síndrome. Para el componente $\mathrm{D}$, este mayor en hombres $(m=8,86)$ que en mujeres. Finalmente, en el componente de RP se indica una prevalencia media en ambos sexos $\mathrm{m}=(35,44)$.

Tabla 5. Prevalencia de Burnout según sexo

\begin{tabular}{lccc}
\hline & Agotamiento emocional & Despersonalización & Realización Personal \\
\hline Femenino & 17,11 & 8,00 & 35,44 \\
\hline Masculino & 16,57 & 8,86 & 35,44 \\
\hline
\end{tabular}

Fuente: elaboración propia

Los datos presentados en la tabla 6 nos muestran que en los tres componentes los docentes de más años de experiencia (21 a 30 años) presentan un nivel bajo de padecimiento del SB. Por otro lado, docentes de menor experiencia ubican dos de los componentes $\mathrm{AE}$ y $\mathrm{D}$ en nivel alto y RP en nivel medio. Los docentes entre 11 a 20 años de experiencia presentan niveles medios de Burnout.

Tabla 6. Prevalencia de Burnout según años de experiencia

\begin{tabular}{cccc}
\hline & Agotamiento emocional & Despersonalización & Realización Personal \\
\hline 1 a 10 años & 20,57 & 10,14 & 34,00 \\
\hline 11 a 20 años & 15,25 & 7,25 & 36,13 \\
\hline 21 a 30 años & 15,34 & 5,00 & 40,00 \\
\hline
\end{tabular}

Fuente: elaboración propia 
Por su parte, la tabla 7 nos dice que en la población de 20 a 30 años de edad todos los componentes se encuentran en nivel medio muy cercanos a la alta (AE $\mathrm{m}=19$; D $\mathrm{m}=9,60$;
RP $\mathrm{m}=34)$. Los docentes en el segmento 41 a 50 años presentan menor índice de padecimiento del SB.

Tabla 7. Prevalencia de Burnout según edad

\begin{tabular}{cccc}
\hline & Agotamiento emocional & Despersonalización & Realización Personal \\
\hline 20 a 30 años & 19,00 & 9,60 & 34,00 \\
\hline 31 a 40 años & 17,50 & 8,33 & 35,17 \\
\hline 41 a 50 años & 14,00 & 7,20 & 37,20 \\
\hline
\end{tabular}

Fuente: elaboración propia

En la tabla 8 se evidencia una tendencia más alta en el componente de $\operatorname{AE}(\mathrm{m}=22,00)$ y baja RP $(\mathrm{m}=33,00)$ para el grupo de personas que laboran de 46 horas semanales en adelante. En cambio, el factor de D ( $\mathrm{m}=$ $9,57)$ es mayor para los que laboran entre 25 a 35 horas semanales.

Tabla 8. Prevalencia de Burnout según horas laborales en la semana

\begin{tabular}{lccc}
\hline & Agotamiento emocional & Despersonalización & Realización Personal \\
\hline 25 a 35 horas & 16,14 & 9,57 & 36,00 \\
\hline 36 a 45 horas & 16,14 & 7,29 & 35,43 \\
\hline 46 horas en adelante & 22,00 & 8,00 & 33,00 \\
\hline
\end{tabular}

Fuente: elaboración propia

El proceso de análisis realizado en las entrevistas con los docentes dio como resultado la emergencia de cuatro categorías centrales, de las cuales emergen subcategorías que permiten interpretar la relación del SB y las PE.

La primera categoría emergente son los síntomas de agotamiento laboral, desde los cuales se desprenden las subcategorías de cambios en la percepción del tiempo, deterioro físico, comportamiento distante con los estudiantes, aislamiento social, mal entendidos y mal genio. La segunda categoría son las PE como detonantes del SB, desde la cual emergen las siguiente subcategorías: pocas actividades extracurriculares, momentos de evaluación a fin de año, actitud de algunos padres de familia y falta de atención de los estudiantes. La tercera categoría emergente son las PE como sistema de afrontamiento. Las subcategorías que la acompañan son rutinas de clase (rito: oración saludo, actividades de motivación contextualización y presentación del tema, evaluación y seguimiento), disposición de los estudiantes, clases divertidas y significativas. La cuarta categoría enunciada son los sistemas de afrontamiento extras a las PE, cuyas subcategorías son escuchar música relajante, lugar tranquilo, oración, hablar con personas alegres, leer y ver una película. 


\section{Conclusiones}

La perspectiva investigativa del SB ha sido abordada desde diversas miradas y caminos epistemológicos y metodológicos, abarcando diferentes profesiones, como médicos, enfermeras, policías, estudiantes, entre otros (Aragón, Morazán y Pérez 2007; Castañeda y García, 2010; Kokinos, 2006; Schaufeli et al., 2002). De esta manera, se ha evidenciado una creciente complejidad en su identificación, tratamiento y correlación, lo que permite identificar los factores de riesgo que la propician y las causas y consecuencias que la acompañan, de tal manera que puedan generarse procesos de intervención que permitan el adecuado afrontamiento de las situaciones que lo producen.

En la literatura investigativa sobre el SB documentada en las diversas bases de datos se evidencia una creciente integración entre el síndrome con diversas variables como son la edad, la cantidad de horas de trabajo, el ambiente laboral; del mismo modo, se ha vinculado con la forma como los colaboradores de las empresas afrontan las situaciones estresantes (Castañeda y García, 2010). Frente a esta literatura y en el ámbito educativo se encuentra un vacío investigativo sobre la relación existente en el SB y las PE en docentes de primaria y secundaria.

Acorde con lo anterior, la presente investigación ha planteado como objetivo identificar la relación existente entre el SB y las $\mathrm{PE}$ en docentes de primaria y secundaria de una institución educativa, analizando el papel del docente reflexivo. Los resultados evidencian que solo un docente $(6,5 \%)$ presenta presencia alta en los tres elementos que componen el SB: AE $\left(\sum=26\right), \mathrm{D}\left(\sum=10\right)$ y RP $\left(\sum=32\right)$. Por otro lado, cabe mencionar que solo un docente aparece con prevalencia baja del SB, presentando en los tres componentes de puntuación ubicados en el percentil bajo AE $\left(\sum=11\right), \mathrm{D}\left(\sum=5\right)$ y RP $\left(\sum=\right.$ 40). Frente a esto, es preciso decir que los docentes que participaron del estudio poseen una prevalencia media del SB, lo que es comprendido como una fuerte tendencia a sufrir sus padecimientos. Dicha prevalencia se evidencian en las tres dimensiones: $\mathrm{AE}$, $M=17,8$; $D, M=9,37 ; R P, M=35,44$. Según Restrepo, Colorado y Cabrera (2006), este padecimiento tendría como principales factores la gran cantidad de horas laborales, el comportamiento agresivo de los alumnos y los conflictos entre profesores, padres y directivas de los colegios, hechos evidenciados también en la codificación de las entrevistas realizadas a los diferentes docentes. Donde la categoría de PE como detonante del SB evidencia la actitud negativa de los padres de familia, la insana presión de los directivos, los momentos de fin de periodo, entre otros, como fundamento de la aparición del Burnout. De la misma manera, Padilla et al. (2009) en su investigación con maestros de Bogotá encontraron que los profesores presentan niveles medios y altos de prevalencia del SB, en relación con los componentes de AE, D y RP.

Comparando las puntuaciones del presente estudio de manera segmentada con cada uno de los factores del MBI, se evidencia que frente al estado civil los docentes solteros son los que más sufren el síndrome, con una prevalencia media en todos los factores, hecho que resulta novedoso frente a otras investigaciones empíricas que evidencian una mayor prevalencia en docentes casados, siendo contrario a estudios como los de Aragón, Morazán y Pérez (2007), Corredor y 
Monroy (2009), Muñoz y Piernagorda (2011), quienes encontraron que las personas que mayormente sufrían el SB son las casadas. Por otro lado, los docente en unión libre son los que menos padecimiento del Burnout padecen, de tal forma que los componentes D y RP se ubican en nivel bajo y el componente $\mathrm{AE}$, en nivel medio. El hecho de que los docentes en unión libre y casados sean los de menor tendencia puede explicarse desde la estrategia de afrontamiento de búsqueda de apoyo social, la cual consiste en acudir a otra persona para buscar ayuda, información, compresión y apoyo emocional (Folkman y Lazarus, 1986; Gálvez et al., 2012).

Frente a la relación del padecimiento del SB, en relación con el sexo, se encontró que el sexo femenino es más propenso a los padecimientos, pues aquí los tres componentes se ubican en el componente medio; por su parte, los resultados para el sexo masculino presentan prevalencia media en D y RP y baja en AE. Este hecho es contrario a estudios como los de Muñoz y Correa (2012) donde la mayor prevalencia estaba en los hombres, y al mismo tiempo concuerda con los resultados encontrados en Portugal donde las mujeres sufren mayores niveles $(34,9 \%)$, mientras que en los hombres fue de 29,0 \% (Gomes, Silva y Moro, 2006).

Por otro lado, la investigación permite identificar que los docentes con menor experiencia son los que más padecen el Burnout. Así, el grupo ubicado en el segmento de edad de 1 a 10 años de experiencia presenta en el factor AE y D niveles altos y en RP, nivel medio. Por el contrario, los docentes más experimentados son los que menos presentan Burnout, de tal forma que los tres componentes en el grupo entre 21 y 30 años de experiencia presentan niveles bajos. Moreno-Jiménez, Garrosa y González (2000); Kokinos (2006), Muñoz y Correa (2013) consideran que la experiencia permite el desarrollo de una serie de recursos que facilitan el proceso de afrontamiento, lo que permite adaptarse de forma más adecuada a los retos, presión y exigencias propios de la profesión docente. Los docentes con menor experiencia afrontan de manera más negativa las situaciones estresantes del aula de clase; de igual forma, existe otro factor y es el ingreso de profesionales sin conocimiento pedagógico y didáctico al sistema educativo colombiano. Los profesionales no licenciados carecen de algunas características formativas que permiten afrontar mejor prácticas educativas.

Se evidenció que los docentes de mayor edad (41 a 50 años) tienen menor tendencia al síndrome, donde la D aparece como medio y el AE y la RP se ubican en nivel bajo. Por el contrario, en los maestros entre los 20 y 30 años de edad, los más jóvenes presentan el mayor padecimiento, donde los tres componentes se ubican en nivel medio. Los resultados son contrarios a los encontrados por Agudo (2004), quien demuestra que existe relación entre la edad y el SB, de manera que a mayor edad mayor Burnout; y son contrarios también a los encontrados por Quevedo (1997), donde el segmento de edad comprendido entre los 35 y 44 años tiene una puntuación media en Burnout, siendo superior al resto, al igual que Cordeiro et al. (2003), donde se encontró que la edad media de 40 años para los docentes es la de mayor padecimiento. Al examinar los resultados a la luz del ciclo vital humano, y según Papalia (2000), encontramos que este 
momento comprende la etapa del ciclo vital denominada adultez intermedia, caracterizada por ser una época de gran productividad, en especial la esfera intelectual, artística, política, y donde se consigue la plena autorización. Es una época de estabilidad de tipo laboral, emocional y psicológico.

En cuanto a la relación del SB con el número de horas de trabajo semanal, la investigación dio como resultado que la prevalencia es mayor en docentes con un rango de horas semanales de 46 o más horas, el cual es identificado con el grupo de mayor horas de trabajo, donde el componente D está en nivel medio y los componentes AE y RP, en nivel alto. Los resultados concuerdan con los encontrados por Aldrete et al. (2002), quienes consideran que el mayor número de horas de trabajo semanal es proporcional a una mayor prevalencia del SB. Por el contrario, el segmento de docentes comprendido entre 25 y 35 horas de trabajo semanal evidenció un padecimiento bajo en $\mathrm{AE} y$ medio en D y RP, siendo el grupo con menor prevalencia. Cabe decir que el número de horas mencionado corresponde a horas de clase y no contempla las horas de preparación y corrección de evaluaciones, lo que hace que la intensidad horaria sea superior.

$\mathrm{El} \mathrm{SB}$, en relación con las $\mathrm{PE}$, presenta un vínculo desde el cual se comprende y afronta la aparición de factores de riesgo, a nivel individual y organizacional. En la primera categoría "síntomas de agotamiento laboral", los docentes experimentan problemas emocionales, físicos y psicológicos; la percepción de que la jornada laboral se hace más extensa: "el día casi no se acaba, la jornada fue mortal" (docente 1). De igual forma, emerge una subcategoría llamada "deterioro físico". En los momentos de mayor tensión laboral los docentes advierten sintomatologías como dolor de hombros, episodios reiterativos de migraña, pérdida parcial y total de la voz, cansancio físico y problemas intestinales.

Uno de los rasgos característicos del SB y las PE se presenta como una subcategoría, "el comportamiento distante con los estudiantes": "hay en algunos grupos muchachitos muy cansones y fastidiosos" (docente 2); "voy contra octavo, llevo listo el machete" (docente 3). De igual forma, el aislamiento social surge como una consecuencia de agotamiento laboral: "es mejor estar solito en mi salón para evitar mal entendidos con los compañeros, a veces son muy chismosos" (docente 4). Finalmente "el mal entendido y mal genio" se presentan de manera regular en el aula, a nivel de directivas, de compañeros docentes y de estudiantes: "El ánimo se disminuye y se responde con apatía, sin amor a la profesión" (docente 5), "los niños y compañeros se vuelven molestos al oído" (docente 6).

El análisis de la segunda categoría, "las PE como detonantes del SB", nos ha proporcionado las subcategorías "pocas actividades extracurriculares fuera del aula": "realizar actividades diferentes, motiva a los estudiantes y con la motivación de ellos la cosa es distinta" (docente 7); "momentos de evaluación fin de año", esta nos refiere que los momentos de mayor desgaste laboral son las entregas de resultados de reporte de estudiantes, ya que los estudiantes y padres de familia ejercen mucha presión sobre el docente; de igual manera, la entrega de evaluaciones finales, reportes a rectoría, hacen una sobrecarga laboral, momento en el que podrían ejercerse actos de intervención. 
Una de las subcategorías más fuertes y recurrentes por los docentes es "la actitud de algunos padres de familia": "los padre de familias creen que somos sus sirvientes y creen que pueden tratarnos como quieran" (docente 8); "los papas creen que el profesor debe enseñar como a ellos les enseñaron” (docente 9); "cuando al niño le va bien es porque es muy inteligente, cuando le va mal, es porque el profesor le tiene bronca" (docente 10). La segunda categoría, "la falta de atención de los estudiantes", es un fenómeno que genera demasiado cansancio en el docente: "perdí 20 minutos de la clase organizando el salón” (docente 11).

En la tercera categoría, "las PE como sistema de afrontamiento", la visión de la clase como un ritual sagrado con unas rutinas de clase ha sido un factor determinante; el estudiante y el profesor conocen su microcosmos y se adecuan al ser de la clase. La disposición de los estudiantes es uno de los factores que permite el afrontamiento del Burnout: "cuando esos muchachos quieren aprenden lo que sea" (docente 12). Esto nos lleva a inferir que el factor motivacional del estudiante, transmitido por el docente, es un factor determinante para evitar el SB; dicho factor va acompañado de la otra subcategoría "las clases divertidas y significativas": "esa clase estuvo tan buena que cuando menos pensé ya se había acabado" (docente 13). Un docente que ama su profesión es capaz de dar significado a lo que enseña.

Finalmente, en la cuarta categoría, "los sistemas de afrontamiento extras a las PE", encontramos una serie de actividades que los docentes reconocen como importantes para afrontar los síntomas del SB surgidos en la escuela; actividades como escuchar música relajante, visitar un lugar tranquilo, tener momentos de oración y encuentro con Dios, hablar con personas alegres, leer y ver una película.

Las PE son el lugar donde el docente desarrolló su profesión, y se convierte en el espacio propicio para el afrontamiento de los factores de riesgo del síndrome. El docente reflexivo encuentra en la innovación de sus prácticas mecanismos de transformación de las situaciones que puedan convertirse en factores de padecimiento de la D, del AE y de falta de RP.

La visión crítica de su propia labor educativa permite la generación de docentes reflexivos (Dewey, 1989; Shön, 2002). De esta manera, el maestro reflexivo se convierte en el punto de partida para comprender las emergencias que faciliten la relación entre SB y PE (Zabala, 2008). Este proceso de transformación y mejoramiento continuo se hace posible gracias a la facultad del docente de transcender el análisis de su práctica, abriendo campo a la composición de una práctica nueva. El docente al visualizar su rol en la práctica logra problematizarla, de modo que a través de la reflexión-acción genera cambios sobre la manera como enseña y sobre cómo aprenden sus estudiantes, aspecto que da paso a la generación de pensamiento reflexivo y se logran así las tres actitudes básicas en un docente reflexivo: mente abierta, referida a la disposición de escuchar puntos de vistas y cuestionar los propios; responsabilidad, entendida como la capacidad de considerar las consecuencias de cada acción, personales, académicas, sociales y políticas; y honestidad, la cual le permite examinar sus propias creencias y concepciones sin justificar su actuación (Dewey, 1989; Kennethy Daniel, 1996). 
De la misma manera, Donald Schön (2002) identifica diversas dimensiones del proceso reflexivo: por un lado, la existencia de una reflexión en la acción y, por otro, una reflexión sobre la acción. La primera se da en la marcha, es un diálogo que se da en solitario en la acción misma; la segunda se da en el diálogo con otros sobre la acción realizada, implica describir o nombrar lo ocurrido. Estos ciclos de reflexión llevan al docente a analizar su ser y su quehacer educativo revelando nuevas imágenes de la práctica. El ser un docente reflexivo implica una actitud de cuestionamiento, de continuo crecimiento, es decir, convertirse en investigador de su propia práctica.

Zeichner (1987) señala que las reflexiones permiten inferir y dar descripciones de las circunstancias personales bajo las cuales se indaga y se toman decisiones sobre la propia práctica. En este sentido, la reflexión es utilizada como un medio de afrontamiento (Connelly y Clandini, 1995). Para Schön (2002) y Perrenoud (2006) los profesionales operan como prácticos reflexivos, es decir, como sujetos que tanto antes y después, como durante su actividad, pueden activar procesos de reflexión sobre la acción, en los cuales involucran el pensamiento intuitivo, el creativo y el racional, lógico y sistemático en beneficio de un proceso educativo menos estresante.

La generación de un pensamiento reflexivo y autónomo que oriente los procesos colectivos de construcción conceptual permite superar el pensamiento rutinario al cuestionar concepciones y prácticas, lo que permite identificar y afrontar aquello que resulta perjudicial para nuestro bienestar laboral. Esta reflexión que afecta el diálogo consciente con uno mismo o con los demás es una estrategia compleja que permite caracterizar y problematizarlos factores de riesgo del SB en contextos educativos diferentes. A partir de estas consideraciones, el desarrollo profesional de los docentes basado en la reflexión sobre sus prácticas como propuestas comunicativas y ético pedagógicas se plantea como una opción formativa que concibe al docente en el proceso como un sujeto activo, participativo, consciente de sus conocimientos y experiencias, y de los obstáculos que pueden presentarle, razón por la cual deben explicitarse y confrontarse. La comprensión y teoría que se pueden elaborar mediante esta reflexión aporta nuevos elementos sobre la experiencia, lo cual permite hacer nuevas interpretaciones de las situaciones y problemas de la práctica en cada contexto.

Finalmente, Reagan (1993) recomienda actividades sobre cómo se puede volver una persona reflexiva, para adquirir un compromiso dinámico e ir más allá de las pautas y conductas rutinarias del quehacer diario. Un buen docente reflexiona individual o colegiadamente sobre su desempeño, hasta ser capaz de volver a su práctica, para hacerla reflexiva, por lo que la esencia del trabajo sobre los estilos pedagógicos busca lo que Bourdieu denomina "habitus" o esquemas que tienen los docentes, para ser transformados a través de procesos de reflexión-acción, reflexión en el desarrollo antes, durante y después de las prácticas docentes.

\section{REFERENCIAS}

Agudo, M. (2004). Burnout y engagement en profesores de primaria y secundaria.

Aguilar, C., Barros, L. y Caro, J. (2011). Variables de la profesión docente asociadas al sindrome de Burnout en colegios de Bogotá 
(trabajo de grado). Universidad de San Buenaventura, Bogotá.

Aldrete, R.M., Pando, M.M., Aranda, B.C. y Balcázar, P.N. (2003). Síndrome de Burnout en maestros de educación básica de Guadalajara. Revista de Investigación y Salud, 5(1), 11-16.

Aragón. M., Morazán D. y Pérez. R. (2007). Síndrome de Burnout en médicos y personal de enfermería del Hospital Escuela "Oscar Danilo Rosales Argüello". Revista Universitas, 2(2), 33-38.

Arata, M. (2008). Burnout, estrés auto-eficacia en maestros peruanos: tres estudios fácticos. Ci Trabajo, 10(30), 1-13.

Balasch, M. y Montenegro, M. (2003). Una propuesta metodológica desde la epistemología de los conocimientos situados: las producciones narrativas. Encuentros en Psicología Social, 1(3), 44-48.

Castañeda, E. y García, J. (2010). Prevalencia del síndrome de agotamiento profesional (Burnout) en médicos familiares mexicanos: análisis de factores de riesgo. Revista Colombiana de Psiquiatría, 39, 67-84.

Connelly, M.y Clandinin, J. (1995). Relatos de experiencia e investigación narrativa. En J. Larrosa et al. Déjame que te cuente. Ensayos sobre narrativa y educación (pp. 11-59). Barcelona: Laertes.

Corbin, J. y Strauss, A. (2001). Bases de la investigación cualitativa. Técnicas y procedimientos para desarrollarla teoría fundamentada. Medellín: Editorial Universidad de Antioquia.

Cordeiro, J. A., Guillén, C. L., Gala, F. J., Lupiani, M., Benítez, A. y Gómez, A. (2003). Prevalencia del Síndrome de Burnout en los maestros. Resultados de una investigación preliminar. Revista Psicología, 7(1). Díaz, F., López, A. y Varela, M. (2012). Factores asociados al síndrome de Burnout en docentes de colegios de la ciudad de Cali, Colombia. Universitas Psychology, 11(1), 217 - 227.

El Sahili, L. y Kornhauser, S. (2010). Burnout en el colectivo docente. México: Escuela Profesional de Comercio y Administración [EPCA].

Dewey, J. (1989).Cómo pensamos. Cognición y desarrollo humano. Barcelona: Paidós.

Escalante Izeta, E.I. (2010). Burnout en docentes: una aproximación a la situación de profesores de escuelas primarias públicas de México (Tesis doctoral). Universidad de Granada, Granada.

Gálvez González, M. Del Águila Hidalgo, B., Fernández Vargas. L., Muñumel Alameda, G, Fernández Luque, C. y Ríos Gallego, F. (2012). Estrategias de afrontamiento ante la muerte del paciente crítico: percepciones y experiencias de las enfermeras. NURE, 10(64).

Gantiva, C., Jaimes, S. y Villa, M. C. (2010). Síndrome de Burnout y estrategias de afrontamiento en docentes de primaria y bachillerato. Psicología desde el Caribe, 26, 36-50.

García, M., Sáez, M. C. y Llor, B. (2000). Burnout, satisfacción laboral y bienestar en personal sanitario de salud mental. Revista de Psicología del Trabajo y de las Organizaciones, 16(2), 381-396.

Gil, P. y Peiró, J.M. (1999). Perspectivas teóricas y modelos interpretativos para el estudio del síndrome de quemarse por el trabajo. Anales de Psicología, 15, 261-8.

Gomes, A. y Silva, M. (2006). Problemas y desafíos en el ejercicio de la actividad docente: un estudio sobre el estrés, "Burnout", y la salud física, la satisfacción laboral de los docentes en $3^{\circ}$ ciclo y secundaria. Revolución Puerto Educación, 19(1), 67-93. 
Kenneth, Z. y Liston, D. (1996). Raíces históricas de la enseñanza reflexiva. En $O b$ servación y Práctica Docente III y IV (pp. 41-50). México: Secretaría de Educación Pública [SEP].

Kokinos, C. (2006). Factor structure and psychometric properties of the Maslach Burnout Inventory-Educators Survey among elementary and secondary school. Teachers in Cyprus. Stress and Health, 22(1), 25-33.

Lazarus, R.S. (1986). Coping Strategies. En S. McHugh, T.M. Vallis (Eds.), Illness behavior. A multidisciplinary model (pp. 303-308). New York: Plenum Press.

Maslach, C. y Jackson, S.E. (1981). Test Manual: Maslach Burnout Inventory. Palo Alto: Consulting Psychologists Press.

Maslach, C. y Jackson, S. (1986). Maslach Burnout Inventory. Palo Alto: Consulting Psychlogists Press.

Moreno-Jiménez, B., Garrosa, E. y González, J. (2000). La evaluación del estrés y el Burnout del profesorado: el CBP-R. Revista de Psicología del Trabajo y las Organizaciones, 16(1), 331-49.

Mora, José. (2000). La necesidad del cambio educativo para la sociedad del conocimiento. Revista Iberoamericana de Educación, 35 .

Moriana, E. y Herruzo, J. (2003). Estrés y Burnout en profesores. International Journal of Clinical and Health Psychology, 3, 597-621.

Muñoz, C. (2012). Relación existente entre las estrategias de afrontamiento y el síndrome de Burnout.XV ${ }^{\circ}$ Congreso Colombiano de Psicología. Colombia.

Muñoz, C. y Piernagorda, D. (2011). Relación existente entre las estrategias de afrontamiento y el síndrome de Burnout en 17 docentes de básica primaria y secundaria de una institución de Cartago, Valle. Revista Psicogente,14(26), 389-402.

Muñoz, C y Correa, C. (2012). Burnout en docentes de primaria y secundaria: perspectiva investigativa en Colombia. Boletín Sociedad Colombiana de Psicología. 29, 7-9.

Muñoz, C. y Correa, C. (2012). Burnout docente y estrategias de afrontamiento en docentes de primaria y secundaria. Revista Colombiana de Ciencias Sociales, 5, 39-55.

Muñoz, C. y Correa, C. (2013). Burnout y estrategias de afrontamiento en docentes. XIV Congreso Virtual Interpsiquis.

Noyola, V. y Padilla, L. (2009). Desgaste profesional (Síndrome de Burnout) en profesores de escuelas primarias públicas del municipio de Aguascalientes. X Congreso del Comie. Veracruz, México.

Padilla, A., Gómez, C., Rodríguez, V., Dávila, M., Avella, C., Caballero, A. y Vives, A. (2009). Prevalencia y características del síndrome de agotamiento profesional (SAP) en docentes de tres colegios públicos de Bogotá (Colombia). Revista Colombiana de Psiquiatría, 38,50- 65.

Papalia, D. (2000). Desarrollo Humano (8va. edición). Madrid: McGraw-Hill.

Perrenuod, P. (2006). Desarrollar la práctica reflexiva en el oficio de enseñar. Barcelona: Grao.

Punceles, A. y Torres, E. (2012).El desgaste profesional (Síndrome de Burnout) en el docente venezolano. $V$ Congreso de Investigación y Creación Intelectual de la UNIMET. Universidad Central de Venezuela,

Ramírez, T., D’Aubeterre, María y Álvarez, Juan. (2010). Características socio-profesionales de los docentes y su percepción de 'estresores' en la Educación Básica en 
Venezuela. Revista de Pedagogía, 31(88), 133-157.

Restrepo, A., Colorado, V. \& Cabrera, A. (2006). Desgaste emocional en docentes oficiales de Medellín, Colombia. Revista de Salud Pública, 8, 63-73.

Rionda, A. y Mares, M. (2011). Burnout en Profesores de Primaria y do Desempeño laboral. Revista Latinoamericana de Medicina Conductua, 2, 43-50.

Reagan, T. (1993). Educating the reflective practitioner: the contribution of philosophy of education. Journal of Research and Development in Education, 26, 189-196.

Salanova, M., Llorens, S. y García-Renedo, M. (2003) ¿Por qué están quemados los profesores? Revista del INSHT, 28, 16-20.

Schaufeli, W. B., Martínez, I. M., Pinto, A. M. Salanova, M. y Bakker, A. B. (2002). Burnout y el compromiso de los estudiantes universitarios: un estudio nacional. Diario de la Cruz - Psicología Cultural, 33, 464-481.
Schön, D. (2002). La formación de profesionales reflexivos. Hacia un nuevo diseño de la enseñanza y el aprendizaje en las profesiones. Barcelona: Paidós.

Unda, S. (2010). Estudio de Prevalencia del Síndrome de Quemarse por el Trabajo (SQT) y su Asociación con la Autoeficacia en Maestros de Primaria de la Ciudad de México. Ciencia y Trabajo, 12, 257-262.

Yslado, R., Núñez, Ll. y Norabuena, R. (2010). Diagnóstico y programa de intervención para el síndrome de Burnout en profesores de educación primaria de distritos de Huaraz e Independencia. Revista de Investigación en Psicología, 3(1) 153-168

Zabala, A. (2008). La práctica educativa. Barcelona: Graó.

Zabala, A. y Arnau, L. (2008). 11 Ideas clave: como aprender y enseñar competencias. España: Graó.

Zeichner, K. (1987). Enseñanza reflexiva y experiencias de aula en la formación del profesorado. Revista de Educación, 282, 161-190. 